\title{
Blending learning: The preferred choice of clinical nurse educators to provide continuing professional development
}

\author{
Antonia Arnaert ${ }^{* 1}$, Hamza Ahmad ${ }^{1}$, Norma Ponzoni ${ }^{1}$, Catherine Oliver ${ }^{2}$, Adriana Grugel-Park ${ }^{1}$ \\ ${ }^{1}$ Ingram School of Nursing, McGill University, Montreal, Canada \\ ${ }^{2}$ McGill University Health Center, Montreal, Canada
}

Received: June 26, 2019

DOI: $10.5430 /$ jnep.v10n1p58
Accepted: August 29, 2019

Online Published: September 24, 2019

URL: https://doi.org/10.5430/jnep.v10n1p58

\begin{abstract}
Introduction and objective: A clinical nurse educators' (CNE) work is primarily focused on ensuring that fellow registered nurses have the skills and training to improve their clinical practice and maintain their professional competence. In recent years, resource limitations and a growing emphasis on self-directed learning have increased the pressure on nurse-educators to integrate e-learning into their teaching methods. While research has evaluated the experiences of nurses on this topic, limited understanding is known of CNEs' experiences. Purpose: This qualitative study explored the CNEs' experiences in facilitating continuing professional development for their nurses and the integration of e-learning in a University Health Center in Quebec, Canada.

Methods: The sample consisted of $7 \mathrm{CNEs}$, who had more than one to 15 years of experience in their current position. Their experiences with e-learning varied: it ranged from incorporating a video-clip in their presentations, to providing input into the learning management system they tested. Semi-structured interviews were thematically analyzed.

Results: Despite participants varied levels of knowledge towards e-learning, all were convinced that this method could be used complementarily alongside hands-on training. Though they recognized the importance of human contact in teaching, they also understood the limitations of the traditional pedagogy; lacking the addition of interactive features. Despite some criticism, CNEs were able to identify opportunities where e-learning could be useful: during nursing orientation, tracking, evaluation and accreditation purposes, content refreshment, and to standardize protocols.

Discussion and conclusions: More research is needed, and cooperative efforts are required from nurses and nurse-management to engage in the promotion of professional development.
\end{abstract}

Key Words: e-Learning, Blended learning, Hands-on training, Clinical nurse educators, Educational technologies, Professional development

\section{INTRODUCTION}

Clinical nurse educators (CNEs) play a significant role in shaping the next generation of nurses. Within a healthcare institution they are responsible for a variety of training and development duties, however much of their work focuses on ensuring that nurses have the skills and training to im- prove their clinical practice and maintain their professional competence. ${ }^{[1,2]}$ The demanding, complex and ever-evolving healthcare system requires nurses to practice a commitment to lifelong learning; whether the nurses are novices or experts in their practice. Although basic principles of nursing care may remain steadfast, nurses must stay informed of evolving

\footnotetext{
*Correspondence: Antonia Arnaert; Email: antonia.arnaert@mcgill.ca; Address: Ingram School of Nursing, McGill University, Montreal, Quebec, H3A 2N7, Canada.
} 
factors, such as: scientific advances and research, evidencebased nursing practice, and to maintain safe and effective patient care. ${ }^{[3-5]}$ Open access to the Internet in the workplace has emerged as a useful tool in promoting evidence-based nursing practice, despite the need for more training in the areas of information and communication technology (ICT), information retrieval and critical appraisal. ${ }^{[6]}$

Creating educational approaches designed to support the learning needs of nurses from pre-licensure through transition into practice and throughout their career, ${ }^{[7]}$ can be challenging for educators. Not only do adult learners engage their particular learning styles, but they also perceive, process, store and recall information differently. ${ }^{[8]}$ In recent decades, CNEs have used conventional learning methods to provide inservices or short courses for hospital unit-based nurses. Faceto-face learning, without the integration of ICT is effective, however, it has been criticized for not recognizing personal differences and learners' needs. In some cases, individual higher-level cognitive skills including problem-solving and creative thinking are disregarded. ${ }^{[9,10]}$ Furthermore, certain barriers in the workplace can inhibit a nurse's participation in hospital-based professional development. Those factors include, working night shifts, experiencing staff shortages, intensified workloads, fatigue or the inability to concentrate after work, and other scheduling or venue conflicts, etc. ${ }^{[11,12]}$ In recent years, resource limitations and the growing emphasis on flexible self-directed learning have increased the pressure on CNEs to integrate e-learning into their teaching methods. ${ }^{[13]}$ E-learning is defined as any educational intervention mediated electronically via the Internet. ${ }^{[14]}$ Research shows that e-learning is gradually becoming a preferred choice when providing professional development due to the benefits associated with it, which include user convenience, program design flexibility, self-pacing and interactivity, adherence to adult learning principles, and accommodation of multiple learning styles. ${ }^{[15-17]}$ The personal convenience of e-learning is highly valued by nurses as it accommodates their working conditions, job demands and personal learning needs. ${ }^{[18,19]}$ The shift towards e-learning will continuously change the way we teach; however, it will also require a re-examination and adaptation of the role of the CNE as a facilitator to foster independent learning rather than providing didactic instruction. ${ }^{[20]}$

Previous research has evaluated nurses' experiences of professional development via e-learning in various clinical environments. Nevertheless, our understanding of the experiences of CNEs regarding the use of e-learning to teach and the difficulties they face when implementing this approach into their curricula requires further assessment. This study aimed to explore CNEs experiences in facilitating continuing professional development for their nurses and the integration of e-learning in a University Health Center in Quebec, Canada. Although the terms e-learning, online learning or web-based learning are often used interchangeably, the concept of e-learning in this study was applied to the use of oriented modules. These were presented on a learning platform or through a software tool, where the interaction and communication between the learner and instructor was entirely online.

\section{Methods}

A qualitative descriptive design was used. After receiving approval from the University Health Center's Research Ethics Board in 2016, a purposive sample of six female and one male CNE was recruited from three hospital sites. A hospitalwide email containing a recruitment flyer was disseminated to all CNEs $(n=34)$ by the senior nurse manager who was responsible for overseeing all nursing professional development activities. Interested participants emailed either the nurse manager or the first author directly and gave their name for the study. Those who expressed an interest were contacted by the first author within 24 hours, more information about the study was provided, an interview was scheduled, and a copy of the consent form was emailed to the participants prior to the interview. At least six months of experience as a CNE was required in order to be eligible to participate in the study. Participants had an average age of 36 years (ranging from 26 to 55), three had a master's degree in nursing and their years of experience as a CNE, varied from approximately one to more than 15 years. The participating CNEs were responsible for the training of nurses on various surgical and intensive care units (ICU). All nurses were comfortable using computers, tablets and emails, and various healthcare technologies in their clinical practice to support the critically ill patients. The CNEs experiences with applying e-learning varied. It ranged from incorporating a YouTube clip in their PowerPoint presentations and the development of specific e-learning modules, to participating in the discussions regarding the selection of the hospital learning management system (LMS). English or French, semi-structured interviews, which lasted approximately 45 to 60 minutes, were conducted by the first and third authors at a convenient time for the participant. Both authors were present for all interviews. All interviews were audio-taped and conducted in a private room on their respective hospital units. The interview guide included questions such as, how do you currently provide professional development to unit nurses? What are the challenges you encounter when offering professional development? What is your opinion about using e-learning to provide professional development 
to nurses? In your opinion, how does e-learning compare to traditional face-to-face professional development? What are the challenges you currently encounter when integrating e-learning? In order to ensure alignment between the study aim and the interview questions, the guide was pilot-tested and validated with key informants, and further refinements were made after the first few interviews. ${ }^{[21]}$ At the start of each interview, the study purpose was re-explained, consent was obtained, and a sociodemographic questionnaire was completed. All interviews were transcribed in the language of the interview and then translated in English for coding purposes. Each transcript was thematically analyzed using the inductive approach, described by Elo and Kyngäs, ${ }^{[22]}$ and supplemented with field notes. A process of open coding was used to assign captions to as many segments of the transcripts as necessary, to describe all aspects of the data. The codes were organized into categories and themes, which captured similar concepts, from which descriptive statements were formed and supported with quotes from the transcripts. This process was repeated until consensus was reached between the first and second authors. Appropriate measures were taken to enhance the trustworthiness of the study. Credibility was established through a process of member checking. To address confirmability, dependability and transferability, the researchers (first and third authors) wrote reflective notes immediately after each interview, documented personal feelings, insights, possible biases and preconceptions, and committed to a detailed description of the research methods, participants, and settings. To respect the anonymity of each $\mathrm{CNE}$, all unit-related information has been kept confidential.

\section{RESUlts}

Study findings revealed three core themes: Diverse Interest and Engagement of Nurses towards Professional Development, Allocation of Resources by Management, and Cultivating Knowledge using a Blended Approach. The participating CNEs in this study found facilitating professional development for their nurses challenging. They believed their primary role was to teach critical-thinking skills. Whereas, the responsibility of nurses and nursing management was to facilitate professional development, and the advancement of nursing knowledge. This triad of CNEs, managers and nurses, is essential to the particular learning environment of each nursing unit. Participants expressed a concern, regarding the nurses' accountability and interest towards further enhancing their clinical knowledge. Some nurses displayed intrinsic motivation to learn, while others did so out of an obligation to obtain the mandatory 20 accredited hours that are prescribed by the Order des infirmières et infirmiers du Québec. ${ }^{[23]}$ According to the CNEs, management on the other hand, appeared to focus on allocating resources towards issues that were unrelated to the education of their nurses. This reduced the provision of diverse learning environments, and as a result the motivation for self-learning. Completing the triad were the CNEs, who were advocating for a combination of hands-on training and e-learning, in the form of a tool, to improve accessibility to information and encourage learner interactivity. Despite the opportunities and advantages of e-learning, participating CNEs said the progression of instructional technology integration, within each of their organizations, was hindered by barriers that restricted newer organizational structures and other formats of learning.

\subsection{Diverse interests and engagement of nurses towards professional development}

Despite their technological savviness, all CNEs used more tangible methods of teaching during their professional development sessions with the unit nurses. Those methods included: traditional in-services, newsletters, workshops, and simulations. However, the most common tool for information dissemination was email. A challenge, CNE participants 2, 3, 4, 5 and 7 (P2, P3, P4, P5 and P7), said they were facing, was that many nurses do not have an institutional email. Some nurses were reluctant to provide their personal email for work-related issues. As stated by P7, "I know a nurse who will not give her email. I naively went to her at the beginning of her shift saying, 'I don't have your email' she was like, 'Well, here is why you don't have my email: I don't want to mix work and outside life', she gave me a whole thing about that". P2 shared a similar, nurse's response, "I got the email, but I don't open it unless it is really pertinent". P3 added, "I am sending a bulk of 110 emails, but I am optimistic if I say, $60 \%$ of them read it. I would say more than $40 \%$ or $50 \%$ depending on the subject. When they like the subject they would probably read it right away". Knowing the low response rate, one of the CNEs, P7 remarked, "If it is something that is really important, I probably would not just want to send it in an email. I would explain it in a staff meeting and make eye contact with the people, to make sure they know it". However, she continued, "If the topic is, for example, concerning a new device that the nurses are required to know, they tend to be eager to learn". She further explained, "Because they are in contact with the device, they have to know... They are afraid of looking like fools, or they are afraid of asking questions. If it is basic stuff, for example using chlorohexidine $0.12 \%$ for a procedure, they won't read it".

However, as P6 stated, "People are running after us for training". P2 further added, "I have units that are very pro-active. 
Nurses will come and ask questions". CNEs P3, P4 and P5, struggled with nurses' intrinsic motivation. For example, P5 explained, "Some people are really frank. They will say, 'I am not interested in reading this at all'". P4 continued, saying that nurses in the past did not ask for relevant articles or information that would demonstrate their desire and eagerness to learn. But, now due to the required accredited hours from the professional order, she said, "I am not sure that they are doing it [requesting information] because they really want to learn. We know this because, some of them sign up for things that aren't even ICU related". Due to these required educational credit hours, $\mathrm{P} 3$ indicated that she has now about $60 \%$ or $70 \%$ attendance [in her training sessions], but P5 stated that, " $40 \%$ of nurses are stars and $60 \%$ are sitting on their behind waiting [for] time to go [by]. I don't know why".

Providing in-service education during working hours for all CNEs is challenging. As elucidated by P7, "[The] ICU can go from being quiet to a Code Blue, and three patients crash and everything is crazy. To have 45 minutes of uninterrupted time is a bit of a stretch". P4 agreed that time is a very precious thing on every unit. "I am often wondering if nurses are really interested [in learning]. When you see them jumping up, when the monitor goes off, they are gone". She understood that nurses cannot be away from their patients for too long. However, she continued, "During the in-service training you don't have their attention either". In the same vein, P2 was concerned that nurses will not retain the information: "They are back and forth doing everything else they have to do... How much [information] are they retaining [from in-service teaching]?" When there was downtime on the unit, P5 explained that this time was used to relax. "It [downtime] is not just [time allotted] to learn because on the ICU it is often a big hurricane". P4 agreed, "We don't see nurses necessarily in their downtime on the unit reading journals, looking up [information], the majority of nurses do not".

Although the CNEs believed that nurses are accountable for their learning, there is an ongoing debate within each of the hospitals sites whether nurses need to be paid for following education at home. P3 shared her mixed feeling about this topic by explaining that, "Coming to a workshop, learning about a new unit protocol, they [nurses] can do it during their work time. But I still think that we as professionals, we have a responsibility to develop our knowledge. I have a problem being paid for that. I read articles at home, I am not paid for it". She continued: "I am not their [nurse] manager, so it is hard for me to say yes or no. I don't have that power". P7 shared a nurse's response: "This is just opening up a can of worms. We do one [e-learning module at home] and there will be a million [of modules] you will expect us to do. It is not fair. We are home". On the other hand, P6 explained that, "They [the nurses] understand and the unions also made it clear, 'We cannot pay for everything'; so, nurses told me that they like it [learning at home]: 'We are in pajamas and it takes us one hour'". For some CNEs like, P2 and P5, the debate; whether to learn at home or at work, is at a stalemate. This is due to a need for an LMS, that is both accessible from home, and enables nurses to track their learning modules and to quiz their understanding. As P2 explained, "An LMS would allow a nurse to access information. So, if we had done IV stuff then a nurse can go home and retrieve the information".

\subsection{Allocation of resources by management}

The CNEs experienced a mixed level of support from their respective management teams in terms of providing and reinforcing professional development on their units. Participants P1, P6, and P7 overall expressed a positive working relationship with their direct management. They stated that management was supportive and encouraged nurses to participate in continuing education. As P7 explained, "It's nice to have a solid support [from management] ... We portray to nurses a united message regarding education". In contrast, four other CNEs, P2, P3, P4, and P5, felt that their management teams did not prioritize or reinforce the continuing education of nurses. As expressed by $\mathrm{P} 4$, "The priorities [of management] are not the nurses learning stuff. It is bringing in this piece of equipment and it's all about budgeting". P5 added, "The middle-management is mainly focused on acquiring beds for the patients". He continued: "Money is the big issue. For example, I asked for a software application that costs about $\$ 30$, they [the management] say no. Even if I buy it, installation is denied". As P2 explained, "If they [the management] are not following up, then I am stuck having to do this [reinforcement] on top of everything else". Despite opposing views regarding management support, all agreed, that they lacked resources, such as: functional computers on the units, had limited internet accessibility, and were unable to access online scientific journals due to subscription cancellations. P1 formulated these limitations as follows, "I use my iPad for teaching because the computers on the units are slow and do not run YouTube". P4 added, "We can access UpToDate but we cannot access PubMed because they [the management] will have to pay for it. Only nurses who have recently graduated have access to online scientific journals".

\subsection{Cultivating knowledge using a blended approach}

Despite the participants' varied levels of knowledge, familiarity and openness towards e-learning, all were convinced that this method could be used complementarily alongside hands-on training. They recognized the importance of hu- 
man contact in teaching. However, they think the traditional face-to-face pedagogy is limited unless interactive features are included. Despite some criticism towards e-learning, all CNEs were able to identify opportunities where this approach could be useful in their practice; more specifically, during nursing orientation activities, tracking, evaluation and accreditation purposes, as content refreshment, and to standardize or harmonize protocols within and across institutions.

\subsubsection{Human element in teaching is irreplaceable}

All CNEs were convinced that e-learning cannot replace the human element. As explained by P3, "We are human beings, we need that personal touch and that one-to-one contact. I called it the human factor". P4 said, "I am older and have been here for a while, I really like the hands-on stuff. Depending on the topic and the personality of the nurse, we will have group or one-to-one sessions". P2 added: "I like to question them [nurses] at the bedside, I like to go in the room and participate in the patient care. For example, when a PICC (Peripherally Inserted Central Catheter) line must be removed, I go over it [the procedure] verbally and then we do it physically". P2 indicated that, "Most of the nurses want hands-on, they want us to make the scenario as realistic as possible". She continued, "You can read something ten times online, but it is not going to change your practice". All CNEs found it challenging to reach the nurses on permanent night shift, however, they exert efforts beyond their regular working hours to sustain contact with them and to reassure their diligence of the protocols. P1 stated, "Especially night nurses, I forget them and I am coming sometimes at night but not every time. I am not spending my nights here". Another said (P2), "I come at 11:00 PM to teach nurses how to fill in the information sheet around epidurals so that I know they are doing it correctly". When it is a new technology, for example, a new intra-aortic balloon pump, P7 said the vendor representative will do the training: "She [representative] will come few times at 7:00 AM to hit the night nurses. Then she comes back at 11:30 AM to hit the day nurses." Due to the interactions with the nurses, $\mathrm{P} 4$ felt that she was a member of the clinical team. However, she expressed a concern that nurses don't call her enough. She stated, "More often, the assistant nurse manager will approach me, saying, 'We are getting a patient from the operating room with a Swan-Ganz catheter. I know that some [nurses] have no experience. Can you be there?"'

\subsubsection{Display creativity to enhance learner interactivity}

In their efforts to engage nurses, all participants were desperately attempting to add various features to their traditional in-person-teaching to increase learner interactivity. As explained by P2, "I always try to make things visual and something different, like finding a YouTube clip. I will actually integrate it into my PowerPoint presentation. I know a lot of people are just more visual, so if they visually see it then they understand it". P3 added, "I try not to do all of it [using all features] because they get bored. So, I try sometimes to have people [clinical domain experts] come in and present instead". P1 went on to explain that she uses her iPad to make her teachings interactive, "I have my personal iPad, which I use to show them videos. They like it because it is different and it is not just me who speaks". One nurse educator, P6 however, stood out by explaining that she uses board games to get nurses engaged in the learning process. She said, "If it is just PowerPoint, people do not like it, so instead I play Jeopardy with the PowerPoint". Some participants, P2 and P5, indicated a willingness to learn how to integrate new features into their PowerPoint presentations, saying "I would do if I could, but I don't know how to do that yet".

\subsubsection{Ambivalence towards the integration of e-learning}

Each CNE in this study approached the new paradigm of integrating e-learning in their practice with varying degrees of enthusiasm and concern. Some embraced the opportunities while others were not yet convinced. P4 articulated it as follows, "There is a push and I almost feel like e-learning is the way to go, but I am not ready to jump on the bandwagon". Other participants, P2, P4, and P7, expressed major criticism towards e-learning. P4 complained about the time it takes to develop an e-module, "It takes two years to develop it [e-modules], and then practice changes before you get it out. How do you keep current?" To increase the use of e-modules, P6, who was part of an educational network for the operating room, emphasized the importance of looking for existing models, "If people have invented something elsewhere, I bring it back to us. We will not reinvent the wheel if something is already well-done". Having been partly involved in the process of developing e-modules, P7 reflected, "I came to realize though that it is pretty difficult to put it [e-modules] together. It is a lot of work". P2 and P4 were unhappy about the length and the user-friendliness of the available e-modules. For example, P4 said, "One e-module on restraints looked interesting, but I never shared it with the nurses because it is too long; the hospital developer should have videotaped herself showing the procedure". P5 shared, that some nurses thought that e-modules developed within their organization were immature in terms of the content displayed. P3, reflecting on the implications of using e-learning to support safe practice, said, "I think there is a good place for technology. It will certainly make part of my work easier. But is it going to improve patient safety?". She continued, "I fear that people will go to e-learning and will not invest any longer in other types of education, for example, reading articles. I fear they will just get satisfied with what is on the 
screen". This CNE made a comparison with the current use of applications in clinical practice, asserting, "The apps are not going to think for you. Critical thinking still needs to be there. It is just a tool."

Despite the criticism and differing experiences, CNEs P1, P2, P3 and P5, identified several opportunities on how e-learning could be integrated and beneficial within their current practice. In regard to the orientation of new nurses on a unit, P1 and P5 indicated that the use of e-modules may reduce the amount of time they spend on training and preparing new staff. P5 emphasized, "Due to the hospital budget cuts, we were obliged to reduce $20 \%$ of our normal orientation time for new nurses. Instead of eight weeks of training it went to six weeks. Using e-learning modules could have mediated this cut". This statement was supported by P3, who added that all protocols and policies should be available online to reduce the costs involved in preparing the nurses for new or existing interventions and techniques. When sending information via email, the CNEs expressed a concern regarding the lack of control or the ability to track and evaluate nurses' knowledge about a given topic. P2 voiced an opportunity to use e-learning to assess nurses' knowledge after an in-service teaching session, "After doing a hands-on physical thing [a procedure], they can go online and complete a quiz using e-learning. Then I know they have actually taken the time to go through the material". P1 emphasized that due to the evaluative component integrated in e-learning modules, it can be used for accreditation purposes. However, modules need to be developed in such a way that the user is encouraged to dedicate time on the material, "You [the nurse] would not receive accreditation if they have not spent at least ten minutes on it [the topic]. So, at least I know, that they have gotten through a certain amount of content". Besides tracking and evaluation, both $\mathrm{P} 2$ and $\mathrm{P} 3$, remarked that e-learning as a tool could support reinforcement and the refreshment of content. P3 explained, "I have a nurse that only works here every two or three months and she often says, 'I don't remember this anymore'. If there was an e-learning module, she could just quickly refresh [her knowledge]". Finally, harmonization of content within and across the affiliated university hospitals would support the standardization of patient care. P3 alleged that, "The same procedure on two different sites [affiliated hospital] is performed differently. What is the best [method] between us? We should not standardize one hundred percent, because our [patient] populations are different".

\section{Discussion}

In a healthcare system that is awash with change, the hospitalbased CNEs have a pivotal role to support both experienced and inexperienced nurses to integrate theory with its applica-

Published by Sciedu Press tion to the workplace; a task that remains extensive and challenging. ${ }^{[24]}$ According to our findings, CNEs experienced various barriers that may impede quality staff education. On the one hand, nurses' lack of accountability and interest to attend professional development due to the absence of allotted time during working hours, obtaining paid or unpaid leave, and use of personal time to undertake mandatory training, ${ }^{[25]}$ and on the other hand the limited resources and organizational support from nursing management. However, with the speed of the digital technology evolution, CNEs in this study saw opportunities on how a blended learning approach with the right tools in place could support their role of promoting evidence-based care while simultaneously balancing the priorities and goals of organizations that are striving to find solutions to achieve efficiency, and ultimately quality and cost-effective patient care. Relating to the findings, two points warrant discussion: (1) the increased need for enfranchising the role of the CNEs, and (2) the opportunities of using a blended learning approach when providing professional development to nurses.

CNEs are saddled with the responsibility of providing education to nurses, however, due to the lack of a line management position within the hospital nursing hierarchy, they don't have the influence or the authority similar to nurse managers who are responsible for overseeing the myriad of daily tasks and challenges related to patient care planning, quality improvement, and allocation of resources. ${ }^{[26,27]}$ Nurse managers understand the importance of facilitating a culture of professional development for nurses within the work environment; ${ }^{[28]}$ however, due to cost cutting measures in hospitals, they are often facing an ethical dilemma on either supporting the organizational goals in terms of cost and productivity or advocating for resources to support professional development for nurses. ${ }^{[29,30]}$ Ominyi and Ezeruigbo ${ }^{[31]}$ added that nurse managers are often hugely constrained by lack of autonomy to mobilize resources for evidence-based nursing practice related activities. This dilemma experienced by nurse managers created a sense of frustration among some CNEs in this study, especially one CNE could not comprehend that the installation of a software application to enhance professional development was denied after purchasing it privately. In combination with a lack of clear identity of their multifaceted responsibilities, it is well-known in the literature that these conflicting situations and a lack of resources for the CNEs to advocate for their specific functions within an organization often result in isolation and erosion of their role. ${ }^{[24,32]}$ Moreover, all of these elements may to lead to a sense of disempowerment. ${ }^{[33]}$ Despite these challenges, other CNEs in this study expressed having a positive working relationship with their nurse managers to develop and 
portray a uniform professional development plan, and felt a sense of belonging with nurses on the unit. Some nurse managers would even ask the CNE to be present on the unit to support the inexperienced nurses when for example a patient return from the operating room with a new type of catheter. Overall, it is imperative that each member of the triad fulfill their responsibilities towards implementing and promoting professional development; however in our digital work environments where e-pedagogy is the future, strengthening this intra-professional relationship will be key as roles and responsibilities may shift and new competencies will be required. Literature exists on the need for a role redefinition of the CNE in light of e-learning as they will need to find new ways to mentor, guide, encourage, and track nurses professional development activities. ${ }^{[20]}$ Some authors have defined a four-fold role of administrator, teacher, developer of educational material, and technical expert; ${ }^{[34]}$ however, more empirical research is needed on how the CNEs role can be adapted to the specific needs of a virtual learning environment. ${ }^{[35]}$

From the CNEs perspective, e-learning can be a solution to resolve the inherent limitations of the traditional face-to-face pedagogy and the increased pressure to provide effective teaching in the current fast-paced and time restricted hospital learning environments. In fact, even the CNEs, who are interested in integrating e-learning into their practice, are faced with infrastructural and organizational limitations. Despite a lack of clear direction from nurse management, all participating CNEs perceived a push to integrate e-learning into their teaching plans. They also favoured a blended-learning approach, as a potential solution for achieving nurses' professional development goals. Literature supports the concept of blended-learning, which is a combination of e-learning and various face-to-face pedagogies. This has been shown to improve content retention, skill acquisition and critical reasoning while maintaining the socialization benefit of face-to-face learning. ${ }^{[36,37]}$ Besides the participating CNEs' preference for this teaching method, Dalhem and Saleh ${ }^{[38]}$ reported that hybrid learning enriches nurses' professional experiences. It is well suited to practical disciplines, ${ }^{[39]}$ like the nursing profession because it fosters skill development. ${ }^{[40]}$

In this study, the results suggested that all CNEs placed a high value on the integration of e-learning strategies. However, none of the participants agreed upon the guiding principles of e-learning. Some saw it as the integration of a YouTube video or a game into their PowerPoint presentations, while others believed it to be the utilization of e-modules available on an LMS; accessible anywhere and anytime. Nonetheless, it was clear that for the majority of this study's participating CNEs, e-learning was thought of as a complementary tool they could use to advance their in-service sessions, specifically in the transmission of theoretical information during one-on-one practice sessions. The concept of combining e-learning with practical training has gained attention in the field of surgical education. ${ }^{[41]}$ Traditionally, surgical skills were taught through expert guidance and feedback, through a mentor-apprenticeship model. ${ }^{[42]}$ However due to the time commitment needed from experienced surgeons and the logistical challenges many hospitals face, new blended learning models are being explored, to create effective and efficient training modules. ${ }^{[4-45]}$ For example, Schmidt and colleagues $^{[45]}$ claimed that their self-directed e-learning approach, consisting of watching the instructional video on laparoscopic suturing and knot tying, three times, prior to every lab session until reaching proficiency allowed surgical trainees to focus more on procedural aspects during operations. Blended learning will become a popular teaching method, however more research is required to assess its pedagogy, efficacy and engagement of the learner. ${ }^{[46]}$

Evidence indicates that the use of e-learning and a blended approach is beneficial for students and educators in academic settings. This qualitative study, however, is among one of the first to provide insight into the experiences of the CNEs in clinical settings. More research is required to further understand this approach from the perspective of a CNE. However, equally important, is understanding managements' perspectives and to evaluate its potential benefits for all stakeholders invested in hybrid learning models. Since the completion of this study, the University Health Center has taken numerous steps towards improving their LMS to increase access to educational material for the professional development of nurses. The current online modules are concise and integrate newly implemented changes in nursing practice. In qualitative research, the inclusion of seven CNEs can be considered a small sample size; however, a point of informational redundancy was achieved in this study. The fact that all participants were either employed in an ICU or surgical setting can be seen as a limitation. Both of these settings typically have a high patient turnover and deal with complex patient care situations that require advanced nursing knowledge, which can lead to similar perceptions and unit based challenges. Findings of this study suggest that all participating CNEs appreciated the integration of e-learning, however they emphasized the importance of retaining traditional styles of teaching, self-motivated, continuing-education for unit nurses, and the engagement of nurse-management in promoting professional development.

\section{CONFLICTS OF INTEREST Disclosure}

The authors declare that there is no conflict of interest. 


\section{REFERENCES}

[1] Hampson J, Gunning H, Nicholson L, et al. Role of clinical practice educators in an integrated community and mental health NHS foundation trust. Nursing Standard. 2017; 32(7): 49-55. PMid:29094540 https://doi.org/10.7748/ns.2017.e10729

[2] Rouleau G, Gagnon MP, Côté J, et al. Effects of e-learning in a continuing education context on nursing care: A review of systematic qualitative, quantitative and mixed studies reviews (protocols). BMJ Open Access. 2017; 7: e018441. PMid:29042394 https ://doi.org/10.1136/bmjopen-2017-01844

[3] Baker JD. Perioperative nurse engagement in lifelong learning. AORN Journal. 2016; 103(1): 3-4. PMid:26746020 https://do i.org/10.1016/j.aorn.2015.11.019

[4] Govranos M, Newton JM. Exploring ward nurses' perceptions of continuing education in clinical settings. Nurse Education Today. 2013; 34: 655-660. PMid:23891123 https ://doi .org/10.1016/ j.nedt. 2013.07 .003

[5] Wellings CA, Gendek MA, Gallagher SE. Evaluating continuing nursing education: A qualitative study of intention to change practice and perceived barriers to knowledge translation. Journal for Nurses in Professional Development. 2017; 33(6): 281-286. PMid:29095214 https://doi.org/10.1097/NND.0000000000000395

[6] Ahmad MM, Musallam R, Allah AH. Nurses and internet healthrelated information: Review on access and utility. CIujul Medical. 2018; 91(3): 266-273. PMid:30093803 https://doi.org/10.1 $5386 /$ cjmed-1024

[7] Gaberson KB, Langston NF. Nursing as Knowledge Work: The Imperative for Lifelong Learning. AORN Journal. 2017; 106(2): 96-98. https ://doi.org/10.1016/j.aorn.2017.06.009

[8] Crannel BA, Witte MM. Clinical practice of registered nurses and learning styles preferences. Institute for Learning Styles Journal. 2012; 1: 1-7.

[9] Johnson SD, Aragon SR, Shaik N, et al. Comparative analysis of learner satisfaction and learning outcomes in online and face-toface learning environment. Journal of Interactive Learning Research. 2000; 11: 29-50

[10] Khatony A, Nayery ND, Ahmadi F, et al. The effectiveness of webbased and face-to-face continuing education methods on nurses' knowledge about AIDS: A comparative study. BMC Medical Education. 2009; 9: 41. PMid:19591678 https://doi.org/10.118 6/1472-6920-9-41

[11] Curtis K, Wiseman T, Kennedy B, et al. Implementation and evaluation of a ward-based elearning programs for trauma patient management. Journal of Trauma Nursing. 2016; 23(1): 28-35. PMid:26745537 https ://doi.org/10.1097/JTN.0000000000 000177

[12] Schneider M, Good S. Meeting the challenges of nursing staff education. Nursing. 2018; 48(8): 16-17. PMid:30045234 https: //doi.org/10.1097/01. NURSE.0000541402.97845.2f

[13] Bloomfield J, While A, Roberts J. Using computer assisted learning for clinical skills education in nursing: integrative review. Journal of Advanced Nursing. 2008; 63(3); 222-235. PMid:18702768 https ://doi.org/10.1111/j.1365-2648.2008.04653.x

[14] Vaono A, Banzi R, Kwag KH, et al. E-learning for health professionals. Cochrane Database of Systematic Reviews. 1(CD011726). 2018. PMid:29355907 https ://doi.org/10.1002/14651858.CD011 736. pub2

[15] Diaz LA, Entonada FB. Are the functions of teachers in e-learning and face-to-face learning environments really different? Journal of Educational Technology \& Society. 2009; 12(4): 331-343.

[16] Gerkin KL,Taylor TH, Weatherby FM. The perceptions of learning and satisfaction of nurses in the online environment. Journal for
Nurses in Staff Development. 2009; 25(1): E8-E13. PMid:19182548 https ://doi.org/10.1097/NND.0b013e318194b6a4

[17] Wu JH, Tennyson RD, Hsia TL. A study of student satisfaction in a blended elearning system environment. Computers \& Education. 2010; 55(1): 155-164. https ://doi.org/10.1016/j.compedu . 2009.12 .012

[18] Karaman S. Nurses' perceptions of online continuing education. BMC Medical Education. 2011; 11: 86. PMid:22013974 https : //doi.org/10.1186/1472-6920-11-86

[19] Yu S, Chen I, Yang K, et al. A feasibility study on the adoption of e-learning for public health nurse continuing education in Taiwan. Nurse Education Today. 2007; 27(7): 755-761. PMid:17175074 https://doi.org/10.1016/j.nedt.2006.10.016

[20] Koch L. The nursing educator's role in e-learning: A literature review. Nurse Education Today. 2014; 34(11): 1382-1387. PMid:24797277 https ://doi.org/10.1016/j.nedt.2014.04.002

[21] Yeong ML, Ismail R, Ismail NH, et al. Interview protocol refinement: Fine-tuning qualitative research interview questions for multi-racial populations in Malaysia. The Qualitative Report. 2018; 23(11): 27002713

[22] Elo S, Kyngäs H. The qualitative content analysis process. Journal of Advanced Nursing. 2008; 62(1): 17-115. PMid:18352969 https://doi.org/10.1111/j.1365-2648.2007.04569.x

[23] OIIQ (Ordre des infirmières et infirmiers du Québec). Norme de formation continue. 2019. Available from: https://www. oi iq. o $\mathrm{rg} /$ formation/norme-de-formation-continue

[24] Coates K, Fraser K. A case for collaborative networks for clinical nurse educators. Nurse Education Today. 2014; 34(1): 6-10. PMid:23628191 https://doi.org/10.1016/j.nedt.2013.04 .003

[25] Coventry TH, Maslin-Prothereo SE, Smith G. Organizational impact of nurse supply and workload on nurses continuing professional development opportunities: A integrative review. Journal of Advanced Nursing. 2015; 71(12): 2715-2727. PMid:26148213 https://doi.org/10.1111/jan.12724

[26] Kodama Y, Fukahori H. Nurse managers' attitudes to promote change in their wards: A qualitative study. Nursing Open. 2017; 4(4): 209 217. PMid:29085647 https://doi.org/10.1002/nop2.87

[27] Skytt B, Ljungren B, Carlsson M. The roles of the first-line nurse manager: Perceptions from four perspectives. Journal of Nursing Management. 2008; 16: 1012-1020. https://doi.org/10.1111/ j.1365-2934.2006.00684.x

[28] Cooper E. Creating a culture of professional development: A milestone pathway tool for registered nurses. The Journal of Continuing Education in Nursing. 2009; 40(11): 501-508. PMid:19904863 https://doi.org/10.3928/00220124-20091023-07

[29] Ganz FD, Wagner N, Toren O. Nurse middle manager ethical dilemmas and moral distress. Nursing Ethics. 2015; 22(1): 43-51. PMid:24477260 https ://doi.org/10.1177/09697330135154 90

[30] Ingwell-Spolan C. Chief nursing offices' views on meeting the needs of professional nurse: How this can affect patient outcomes. Healthcare. 2018; 6: 56. PMid:29857471 https://doi.org/10.3390/ healthcare 6020056

[31] Ominyi J, Ezeruigbo CFS. How nurse manager's position in the hospital hierarchy influences evidence-based practice implementation in nursing: A qualitative case study of the Nigerian acute care setting. Journal of Nursing Education and Practice. 2019; 9(6). https ://doi.org/10.5430/jnep.v9n6p14

[32] Malik G, McKenna L, Plummer V. Perceived knowledge, skills, attitude and contextual factors affecting evidence-based practice 
among nurse educators, clinical coaches and nurse specialists. International Journal of Nursing Practice. 2015; 21: 46-57. PMid:25355492 https://doi.org/10.1111/ijn.12366

[33] Popa M. An integrative review of the role, challenges, and facilitators of clinical nurse educators in labor and delivery units. Master of Science in Nursing, The University of British Columbia, Canada), Unpublished Work. 2018.

[34] Williams PE. Roles and competencies for distance education programs in higher education institutions. American Journal of Distance Education. 2003; 17(1): 45-57. https://doi.org/10.1207/S1 5389286AJDE1701_4

[35] Bennet S, Lokyer L. Becoming an online teacher: Adapting to a changed environment for teaching and learning in higher education. Educational Media International. 2004; 41(3). https : //doi .org/ 10.1080/09523980410001680842

[36] Rowe M, Frantz J, Bozalek V. The role of blended learning in the clinical education of healthcare students: A systematic review. Medical Teacher. 2012; 34: e216-e221. PMid:22455712 https: //doi.org/10.3109/0142159X.2012.642831

[37] Suliman S, Hassan R, Athamneh K, et al. Blended learning in quality improvement training for healthcare professionals in Qatar. International Journal of Medical Education. 2018; 9: 55-56. PMid:29478042 https://doi.org/10.5116/ijme.5a80.3d88

[38] Dalhem WA, Saleh N. The impact of eLearning on nurses' professional knowledge and practice in HMC. Canadian Journal of Nursing Informatics. 2014.

[39] Facharzt NM, Abos KIK, Algadi S, et al. Blended learning as an effective teaching and learning strategy in clinical medicine: a comparative cross-sectional university-based study. Journal of
Taibah University Medical Sciences. 2013; 8(1): 12-17. https: //doi.org/10.1016/j.jtumed.2013.01.002

[40] Manyazewal T, Marinucci F, Belay G, et al. Implementation and evaluation of a blended learning course on tuberculosis for front-line health care professionals. American Journal of Clinical Pathology. 2017; 147: 285-291. PMid:28395055 https ://doi.org/10.109 3/AJCP/AQX002

[41] Larvin M. E-learning in surgical education and training. ANZ Journal of Surgery. 2008; 79: 133-137. PMid:19317777 https : //doi.org/10.1111/j.1445-2197.2008.04828.x

[42] Gallagher AG, O'Sullivan GC. Fundamentals of surgical simulation: principles and practice. Springer, Berlin. 2011.

[43] Jayakumar N, Brunckhorst O, Dasgupta P, et al. e-Learning in Surgical Education: A Systematic Review. Journal of Surgical Education. 2015; 72(6): 1145-1157. PMid:26111822 https ://doi .org/10.1 $016 / j \cdot j$ surg. 2015.05.008

[44] Maertens H, Madani A, Landry T, et al. Systematic review of elearning for surgical training. British Journal of Surgery. 2016; 103(11): 1428-1437. PMid:27537708 https ://doi .org/10.100 2/bjs. 10236

[45] Schmidt MW, Kowalewski KF, Trent SM, et al. Self-directed training with e-learning using the first-person perspective for laparoscopic suturing and knot tying: a randomised controlled trial. Surgical Endoscopy. 2019.

[46] Coyne E, Rands H, Frommolt V, et al. Investigation of blended learning video resources to teach health students clinical skills: An integrative review. Nurse Education Today. 2018; 63: 101-107. PMid:29425738 https://doi.org/10.1016/j.nedt. 2018.01 .021 